\title{
LE PENICILLIUM CAMEMBERTI ET LES ORIGINES DU CAMEMBERT
}

\author{
par \\ M. DESFLEURS \\ Ingénieur chimiste I.C.L., Docteur ès sciences $3^{\mathrm{e}}$ cycle \\ Laboratoire de Microbiologie \\ Centre de $3^{\mathrm{e}}$ cycle de Sciences du Lait et Centre du Lait et des Viandes \\ de l'Université de Caen
}

La plupart des fromages français ont une origine très ancienne qu'il est souvent difficile de prouver quand on n'en retrouve pas mention dans les archives. Si l'on sait que le Brie existait déjà du temps du due Charles d'Orléans, père de Louis XII, que l'Augelot, appelé actuellement Pont-l'Evêque, date du Moyen Age, pour son voisin le Camembert, fabriqué lui aussi à ses débuts dans le Pays d'Auge, à ma connaissance, on ne peut remonter aussi loin. On en attribue généralement la création à Marie Harel, lors de son établissement dans sa ferme de Camembert en 1791. Les choses ne sont pas aussi simples.

Thomas Corneille mentionne dans son Dictionnaire (1708) que le fromage de Camembert était déjà vendu en 1702 sur les marchés de Vimoutiers et d'Argentan (Orne). La fabrication en était faite par les fermières de la région. Rien done de plus naturel que Marie Harel, née Fontaine, se mit à en produire dans sa ferme de Camembert.

Marie Catherine Fontaine naquit à Crouttes (Orne) le 28 avril 1761, fut baptisée le même jour par le vicaire Le Belhomme de cette paroisse, en présence de son parrain Olivier Legendre et de sa marraine Marie Fontaine, femme de Germain Vas, ses oncle et tante, tous deux de Camembert [2]. Elle était la seconde de dix enfants qu'eurent ses parents : Jacques Etienne Fontaine, né à Camembert le 25 mai 1737, décédé à Saint-Gervais le 6 février 1820 et Marguerite Legendre, décédée à Camembert le 12 octobre 1782, qui s'étaient mariés à Saint-Léger, le 28 octobre 1758. Parmi leurs 4 garçons et 6 filles, 7 sont nés à Crouttes et 3 à Camembert.

Marie Catherine Fontaine épousa le 10 mai 1785, à Camembert, Jacques Harel, fils majeur de Charles Harel, laboureur, et de Marie Leloup, de Guerquesalles (Orne). La bénédiction nuptiale leur fut donnée par J. Pichonnier, curé de Camembert [2]. 
Les époux Harel exploitèrent en 1791 la ferme de Beaumoncel, à Camembert (Orne). Ils y cachèrent un prêtre réfractaire, venant de la Brie, pendant les heures sombres de la Révolution. Ce fut cet ecclésiastique qui donna d'utiles conseils à Marie Fontaine, épouse Harel, d'après son petit-fils Victor Paynel [1], pour la fabrication de ses camemberts, qu'elle mit au point et perfectionna. Le succès venant, elle vendit ses fromages sur place, à Vimoutiers et aussi au marché d'Argentan où, en 1798, elle établit un débit chez une dame Trouvé, marchande de comestibles, rue de l'Horloge [10].

Sa fille aînée, Marie Harel, née à Roiville, le 29 décembre 1787 [2] mariée en 1813 à Augustin Thomas Paynel de Champosoult et décédée le 14 mai 1855 à Champosoult, continua les fabrications de sa mère. Elle reçut des encouragements et des récompenses, en particulier de l'Association Normande en 1846. Augustin Thomas Paynel vendit, par l'intermédiaire de Mme Chalange, marchande de fromages, rue de la Monnaie, ses camemberts à Caen.

Ce sont les descendants de Marie Harel-Paynel, qui donnèrent de l'extension à la fabrication du camembert $[10,13]$ : MM. Paynel aîné, à Grandchamp (Calvados), Paynel Cyrille, au Mesnil-Mauger (Calvados), Paynel Victor, à Champosoult (Orne), Mme Paynel Julie, femme Lebret, à Mézidon (Calvados), auxquels il faut ajouter Mme Morice, de Lessard, qui était la filleule de M. Paynel père et qui aurait fondé dans le Calvados la première fabrique de camemberts.

M. Victor Paynel a d'ailleurs raconté comment il fut à la base de la diffusion de ce fromage [1].

Napoléon III étant venu à Surdon en 1856 inaugurer un tronçon de la voie ferrée Paris-Granville, M. V. Paynel eût l'idée de lui offrir des camemberts. L'empereur les ayant appréciés, demanda à M. Paynel de lui en porter aux Tuileries, ce qu'il fit. Ensuite, il dut en expédier. En bon Normand, il déclara que cela lui avait coûté cher, mais qu'ensuite, il avait eu du mal à satisfaire les commandes.

On peut évaluer la dépense, en ce qui concerne les fromages, si l'on admet que leur prix était déjà celui que donne le Professeur Pouriau [13], quelque vingt-cinq ans plus tard, c'est-à-dire 5 francs la douzaine en été, 8 à 9 francs en automne et en hiver, soit 7 francs en moyenne. Les bons camemberts se vendaient 0,90 à 1 franc pièce dans les maisons de $1^{\mathrm{er}}$ ordre, à Paris. Il est vrai que vers 1880, il s'en fabriquait aussi avec du lait partiellement écrémé, vendus 60 à 70 eentimes pièce au détail, différence de prix justifiée par la qualité.

Quoiqu'il en soit, il semble bien que la fabrication du camembert, d'après ce qui précède, ait été localisée à ses débuts dans la région de Camembert et Vimoutiers. Son extension première à d'autres 
régions de l'Orne et du Calvados [12] n'a pas été le fait de la seule famille de Marie Fontaine, femme Harel, mais on comprend bien pourquoi on lui a érigé une statue à Vimoutiers et une stèle à Camembert.

La moisissure qui recouvrait les camemberts était alors le Penicillium camemberti, appelé à l'époque $P$. album. Elle est décrite dans les traités de laiterie du Professeur Pouriau [13], de Bochet [3], comme couvrant les fromages d'une couche blanche, laineuse, qui, après plusieurs jours, se teinte de bleu clair, coloration qui fonce par la suite. C'est le propre du $P$. camemberti. Le Professeur Pouriau décrit le même champignon pour les fromages de brie, de coulommiers, de neufchâtel (bondon). Pour ce dernier, étant donné la présence spontanée actuelle de $P$. caseicolum $=P$. candidum dans les caves de la région de Neufchâtel, je pense qu'au moins certains de ces fromages étaient alors aussi recouverts de cette moisissure blanche et qui le reste, comme c'était également le cas pour les bries et coulommiers [16].

Le nom de $P$. album fut d'abord donné par Preuss [14] au $P$. camemberti. C'est ainsi que l'appelle Mazé [11], dénomination souvent conservée en France. Ces deux auteurs n'en donnent pas de description adéquate. De même, pour Johan-Olsen [9] qui l'appelle $P$. aromaticum $I I I$ ou $P$. aromaticum casei $I I I$. En 1904, Thom [18] l'isole sur un Camembert français et profitant de ce qu'il n'a pas été correctement décrit, il le baptise $P$. camemberti Thom, en en donnant la spécification. Il confirme ce nom en 1910 dans son étude [19], puis dans son livre [20]. Raper et Thom le reprennent dans leur manuel [15]. Sopp l'appelle $P$. camembert dans sa Monographie [17].

Le type de l'espèce est représenté par la souche NRRL 877 que Thom isola en 1904 et également la NRRL 878 qui provient de la collection de Biourge. Depuis cette date, la souche NRRL 877 est restée sans changement, tant dans son apparence que dans sa structure, ainsi que le soulignent Raper et Thom. M'étant procuré cette souche, j'ai voulu vérifier le fait et l'ai cultivée parallèlement avec un exemplaire de $P$. camemberti isolé sur un fromage de chèvre de Saint-Marcellin. Mes observations [5] ont confirmé l'absence de variation pendant cette soixantaine d'année de culture continue, fait qui mérite d'être signalé, car peu fréquent chez les Penicillia. J'ai trouvé simplement que la souche NRRL 877 s'accroissait un peu moins vite que la souche Saint-Marcellin, ce qui était normal, s'agissant d'une souche de collection repiquée moins fréquemment. Depuis d'ailleurs, des cultures plus rapprochées ont rendu les vitesses de pousse sensiblement égales.

Cela ne veut pas dire, cependant, que l'on ne puisse rencontrer de mutants. Raper et Thom citent le cas de la souche NRRL 1740 reçue du Professeur Hastings de l'Université de Wisconsin. Elle diffère du type en donnant une colonie plus lâche, moins serrée, 
des conidiophores généralement plus rugueux et une couleur se rapprochant du tilleul-chamois, sans vert bleuté.

Il faudrait, peut-être, y joindre le Penicillium que Drewes [6] dit avoir trouvé sur des fromages allemands, qui différerait du $P$. camemberti, pour lequel il propose le nom de $P$. henebergi, mais sans donner de description valable.

Le $P$. camemberti est incontestablement la moisissure primitive du camembert. Sa fabrication, d'abord uniquement fermière, devint artisanale, puis avec le succès, industrielle, en même temps que son aire de production s'étendait, ce qui était facilité par l'absence de l'appellation contrôlée "Camembert" cadeau des Normands à l'industrie fromagère et pas uniquement française. La fabrication en quantité rendait plus difficile et aléatoire la propagation de la moisissure par l'ambiance de la fromagerie. Les fromagers durent avoir recours à l'ensemencement artificiel. Les producteurs de ferments fournissant plus volontiers une suspension de conidies de $P$. caseicolum, l'aspect plus agréable d'un fromage bien blanc, peut-être aussi le goût de la clientèle évoluant vers des camemberts plus doux et moins coulants, firent que le $P$. caseicolum supplanta totalement le $P$. camemberti.

Si je me souviens parfaitement d'avoir vu, au début de ce siècle, des Camemberts recouverts d'une moisissure gris vert très clair, il y a longtemps que l'on ne trouve plus le $P$. camemberti que sur quelques fromages de chèvre et encore y est-il ensemencé.

La souche type NRRL 877 provient d'un camembert français. J'ai lieu de penser que la souche que j'ai étudiée, venant d'un SaintMarcellin, pouvait y avoir été ensemencée. Je n'ai pas trouvé ces vingt dernières années de $P$. camemberti provoquant des accidents de "bleu" sur les fromages. Il est vrai qu'ils sont devenus moins fréquents depuis les travaux qui ont été faits sur le sel sous la direction de M. le Professeur Jacquet [7, 8]. Comme cette moisissure n'est pas signalée en dehors de l'industrie fromagère et que celle-ci ne l'emploie plus du tout en Normandie, on pouvait se demander si elle existait encore à l'état naturel et spontané.

Un concours exceptionnel de circonstances allait me permettre de répondre affirmativement à cette question.

Au début de l'automne 1967, je fus appelé par Mlle G. Fontaine, qui avait une pousse anormale affectant la surface des livarots qu'elle fabrique dans sa fromagerie de Vimoutiers. (Mlle Fontaine est une descendante de l'un des frères de Marie Fontaine, épouse Harel) (1).

Devant les ennuis qu'auraient pu provoquer ce développement causé par un microorganisme apparenté au groupe des levures,

(1) Je remercie bien vivement M. Fontaine-BLanchon, père de Mue Fontaine à l'amabilité duquel je dois la presque totalité des documents eoncernant Marie HaRel. 
nous décidâmes, puisque nous en avions la possibilité, d'arrêter la fabrication et de procéder à la désinfection intégrale de tous les locaux, sans exception, par le formol. A la suite de quoi, avec l'aide de quelques ferments spécifiques du livarot, la fabrication reprit sans donner aucun ennui.

Au bout d'un mois et demi environ, e'est-à-dire vers la minovembre, Mlle Fontaine me signala l'apparition sur ses fromages d'une moisissure blanche, feutrée, laineuse, qu'elle pensa être du $P$. caseicolum. Cette pousse était de peu d'importance, puisque le lavage des livarots supprimait tout mycelium. Cependant, si ce lavage tardait, de blanc, le champignon mieroscopique avait tendance à bleuir.

Je fis des prélèvements, tant des parties blanches que des bleues, pour constater, après isolement, cultures, mesures, qu'il s'agissait uniquement du $P$. camemberti. En voici d'ailleurs les caractéristiques principales, telles que je les ai déterminées :

Sur milieu de Czapek gélosé, la colonie de cette souche a un diamètre de $15 \mathrm{~mm}$ au $5^{\mathrm{e}}$ jour ; 30 au $10^{\mathrm{e}}$ et $40 \mathrm{au} 15^{\mathrm{e}}$ jour après l'ensemencement à la température de $20^{\circ} \mathrm{C}$. Elle pousse d'abord blanche, très laineuse et épaisse. Elle a tendance à bleuir au centre vers le $6^{e}$ jour, teinte qui devient bleu verdâtre pastel dans les jours suivants, pour ensuite devenir bleu-verdâtre-gris très pâle et finalement se teinter légèrement de chamois. En résumé la colonie s'accroît avec un bord net, sans marge, blanc, avec projection dans le sens de l'accroissement d'hyphes infertiles, ni réunis en faisceaux, ni funiliformes. En allant vers le centre on trouve une teinte bleutée, puis bleu-gris verdâtre pâle, qui devient tilleul au centre un peu surélevé. Ces couleurs ne forment pas de zones nettes. L'aspect est laineux, bien sporulé et très touffu. Des sillons radiaires prennent naissance après la partie centrale surélevée. Il n'y a pas d'exsudat. Le revers est blanc un peu jaunâtre, tirant vers le chamois au centre, sillonné en rayons.

Les conidiophores se dressent directement du substrat ou sont souvent disposés comme des branches sur le mycelium aérien, donc de longueur variable, allant jusqu'à $600 \mu$ pour les plus longs, pour une largeur de 3 à $4 \mu$. Ils sont généralement simples, peu ramifiés, assez peu rugueux, de même les articles du pinceau qui les termine. Ce dernier est asymétrique, non divergent. Les branches sont disposées irrégulièrement, de longueur 12 à $18 \mu$ pour une largeur de $3 \mu$ environ. Les métules, souvent peu différeneiées des branches, sont à des niveaux variables, généralement en groupe de 2 à 3 , longues de 10 à $14 \mu$ larges de $2,5 \mu$. Elles portent des verticilles de 3 à 5 stérigmates, de 10 à $14 \mu$ de long sur 3 de large. Ces phialides se terminent par des chaînes de conidies qui s'emmêlent pour former souvent des amas plus ou moins en boule (fig. 1). Quand les conidies se forment, elles sont ovales, mais en mûrissant deviennent grossièrement sphériques avec un diamètre 
de 3,5 à $4,5 \mu$. Elles sont peu colorées, leur paroi est lisse au microscope électronique (fig. 2).

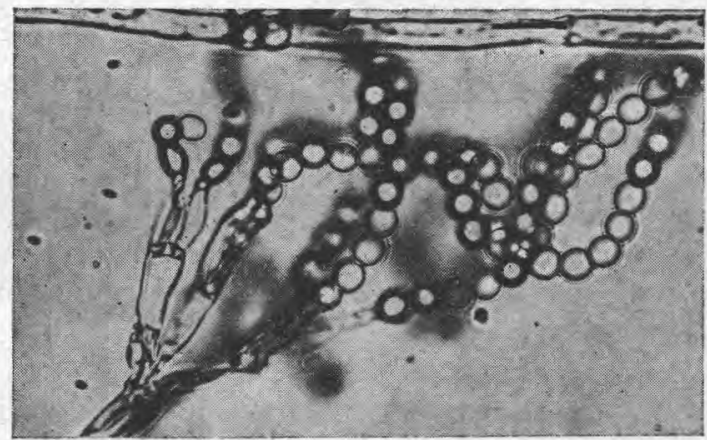

Fig. 1. - Pinceau du P. camemberti, souche Vimoutiers.

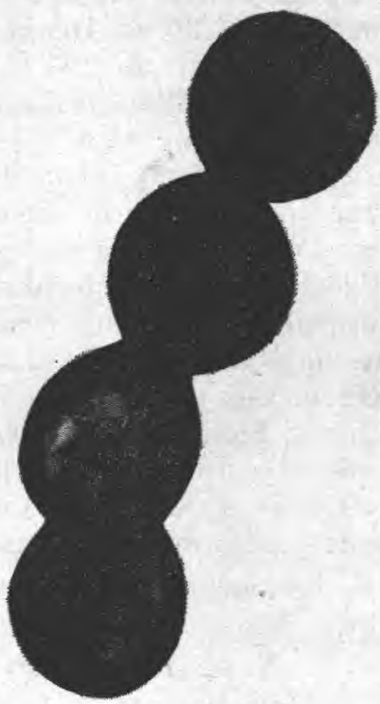

Fig. 2. - Aspect au microscope électronique des conidies du $P$. camemberti, souche Vimoutiers.

Ce Penicillium se classe donc dans la section des Asymmetrica, groupe des Lanata. Sa description correspond à celle de Raper et Thom pour le $P$. camemberti et à celles que j'en ai moi-même données [5]. Il en est de même sur le milieu de Czapek peptoné gélosé et milieu au malt, glucosé, peptoné et gélosé, employés pour la reconnaissance des Penicillia.

Je signale, cependant, que sur milieu de Czapek le centre de cette souche prend une couleur tilleul, ce qui la différencie des 
souches NRRL 877 et Saint-Marcellin, mais la rapproche de la NRRL 1740. C'est, d'ailleurs, le seul point de ressemblance, car notre souche présente les couleurs gris bleu-verdâtre pâle habituelles et est très touffue et extrêmement sporulée, beaucoup plus que la NRRL 877 et Saint-Marcellin. Il en résulte que la teinte des colonies est accentuée sur tous les milieux, puisque les conidies sont seules colorées.

Cette abondance de fructifications est généralement la règle pour une souche non cultivée et venant d'être isolée. C'est le seul moyen de défense et de propagation de l'espèce. Les cultures successives diminuent habituellement la sporulation.

Je pense qu'on peut considérer cette souche de $P$. camemberti comme type et d'une lignée pure, puisque parvenue jusqu'à nous en dehors de la propagation sur les fromages, par les moyens propres aux moisissures qu'il serait intéressant de pouvoir préciser.

De quelle façon ce $P$. camemberti a-t-il été amené sur les livarots puisqu'il faut exclure la contamination par les locaux ?. Três vraisemblablement par le lait lui-même, trait dans les prairies, par l'air, par le personnel, peut-être par les emballages!

Si l'on se réfère à une note présentée à l'Académie d'Agriculture par le Professeur R. Heim [4], à certaines époques de l'année, l'air est beaucoup plus chargé en spores de moisissures et l'automne est de celles-là. Les fromagers les connaissent bien, car les accidents de "bleu " y sont plus fréquents. Mlle G. Fontaine m'a d'ailleurs signalé que ce $P$. camemberti apparaît à peu près régulièrement tous les ans sur ses livarots, principalement à l'automne.

Quoiqu'il en soit, Vimoutiers étant à $5 \mathrm{~km}$ de Camembert, il me paraissait intéressant de signaler la persistance du $P$. camemberti dans la région d'origine du camembert.

Depuis, je dois à l'obligeance d'un industriel fromager du Pays d'Auge, que je tiens à remercier vivement, d'avoir pu ensemencer avec ce $P$. camemberti des camemberts fabriqués, les uns avec du lait cru, les autres avec du pasteurisé. Par rapport aux témoins recouverts de $P$. caseicolum, les fromages obtenus avaient un goût très fin, plus prononcé, agréable, rappelant un peu le chèvre.

\section{Résumé}

Après avoir indiqué quelles sont les origines du camembert et la part prise par Marie Harel et sa famille, dans sa fabrication, M. Desfleurs explique comment il a isolé le $P$. camemberti qui persiste dans la région de Camembert et en donne la description.

\section{Summary}

Stating the origin of the camembert cheese and the part that took Marie Harel and family in its fabrication, M. Desfleurs explains 
how he discovered the $P$. camemberti which is still to be found in the Camembert area. He gives its description.

\section{BIBLIOGRAPHIE}

[1] Agriculture et Elevage dans l'Orne. No 16, juillet-août 1957.

[2] Archives paroissiales.

[3] Bochet (L.). Manuel pratique de l'industrie laitière. Fritsch Z., éd., Paris, 1895 .

[4] Charpin (J.), Charpin (H.) Mme, Aubert (J.), Renard (M.) Mie, LAURTol (M.) Mme. Sur le recensement atmosphérique des spores de moisissures. C. R. Ac. Agric. de France, $\mathrm{n}^{\circ} 14,19$ octobre 1966.

[5] Desrleurs (M.). Contribution à la connaissance du genre Pericillium. Application à la fabrication des fromages à pâte molle et notamment du camembert. Thèse $3^{\mathrm{e}}$ cycle, Fac. des Sc. de Caen, 10 juin 1966.

[6] Drewes (K.). Die Reifungspilze des Sauermilchkäses unter besonderer Berucksichtigung ihres Caseinablauvermögens. Milchs. Forsch, 18, 289-330, 1937.

[7] Jacquet (J.), Villette (O.) Mme, Delacroix (J.), Gondouin (H.) et Desfleurs (M.). Considérations sur l'action du $p \mathrm{H}$ dans la croissance des moisissures utilisées pour la fabrication des camem berts. Rôle du sel. Bull. Soc. Linn. Norm., 9e série, 8, 1954-55, 115 . 133.

[8] JACQUET (J.). Les maladies des fromages simples curiosités ou brar, the véritable de la pathologie comparée. Rev. Path. Générale et de Physiol. clinique, $\mathrm{n}^{0}$ 742, novembre 1962, 1007-1044.

[9] Johan-Olsen (O.). - Die bei der Käsereifung wirksamen Pilze. Centbl. F. Bakt, etc. (II), 4, 161-169, 1898.

[10] $J$. de Normandie, n⿳0 282, 28 octobre 1940.

[11] Mazé (P.). Les microbes dans l'industrie fromagère. Ann. Inst. Pasteur, 19,1905 .

[12] MorIÈre (J.). - Industrie fromagère dans le Calvados, 1877.

[13] Pouriau (A. F.). La Laiterie. Audot, Lebroc \& Cie, édit., 8, rue Garaneière, Paris, 3e éd., vers 1882.

[14] Preuss (G. T.). - Uebersicht untersuchter Pilze, etc., Linnaea, 24, $25,26,1851-1853$.

[15] Raper (K.) et Thом (C.). A Manual of the "Penicillia". Baillière, Tindall et Cox, édit,, Londres, 1949.

[16] Roger (G.). Fabrication des fromages de Brie par l'emploi de ferments naturels et des spores de Mucédinées. Com. à la Sté d'Agr. de Meaux, 19 novembre 1898.

[17] Sopp (O.). Monographie der Pilzgruppe Penicillium. Videnskapselkapets Skrifter. I Mat. Naturv. Klasse, no 11, 1-208, 1912.

[18] Тном (C.). Fungi in cheese ripening. U.S. Dept. Agr.-Bur. Anim. Ind. Bul., 82, 1906.

[19] Тном (C.). Ibid., bull. 1, 1910.

[20] Тном (C.). The "Penicilla", Williams \& Wilkins Co, édit., Baltimore, 1930. 